\title{
Resource Allocation for Multipoint-to-Multipoint Orthogonal Multicarrier Division Duplexing
}

\author{
Poramate Tarasak ${ }^{\dagger}$ and Hlaing Minn ${ }^{\ddagger}$ \\ $\dagger$ Institute for Infocomm Research, Agency for Science, Technology and Research (A*STAR) \\ 1 Fusionopolis Way, \#21-01 Connexis, Singapore 138632, \\ $\ddagger$ University of Texas at Dallas, Richardson, TX, USA, \\ Email: ptarasak@i2r.a-star.edu.sg, hlaing.minn@utdallas.edu
}

\begin{abstract}
Orthogonal multicarrier division duplexing (MDD) is a new duplexing scheme that gains the advantages of both time division duplexing and frequency division duplexing. Resource allocation for multipoint-to-multipoint (M2M) MDD is considered in this paper. We propose joint subcarrier and power allocation for M2M MDD that maximizes the sum rate of the network under either total or individual power constraint. Simulation results show that MDD outperforms conventional TDD and TDDOFDMA systems. Some insights on M2M MDD under different channel conditions are given and convergence of the proposed algorithm is shown through simulation results.
\end{abstract}

\section{INTRODUCTION}

Multipoint-to-multipoint (M2M) communications allows three of more users to exchange information. Typical applications of M2M communications include conference call and video conference. Due to surging in multimedia contents, M2M communications is growing and has been applied to applications such as content delivery networks [1] and peerto-peer networks [2].

Multicarrier communications lies at the heart of current and future wireless communications systems. Orthogonal frequency division multiplexing (OFDM), the most popular multicarrier communication technique, has been adopted in many well-known standards such as IEEE802.11 (Wi-Fi), IEEE802.16 (WiMAX) and LTE [3]. The advantages of OFDM include robustness to intersymbol interference, simple channel equalization, relaxed symbol timing requirement, frequency diversity through scheduling on orthogonal frequency division multiple access (OFDMA).

Duplexing refers to the separation of transmission direction between two terminals. Time division duplexing (TDD) and frequency division duplexing (FDD) have been fundamental duplexing schemes in wireless communications [4]. In TDD, the transmission in each direction occupies the whole frequency band and each direction uses different time slots. In FDD, the transmission in each direction occupies different parts of bandwidth but the transmissions in both directions can occur simultaneously. While TDD enjoys full frequency diversity from using the whole bandwidth, there is a required guard interval separating the transmission in each direction, accounting for round-trip propagation. On the other hand, FDD enjoys full duplex transmission but there is a need for a guard band, accounting for possible interference. Both guard interval and guard band effectively reduce the transmission rate as they occupy certain amount of resource.

Recently, orthogonal multicarrier division duplexing (MDD) was proposed to gain the advantages of both TDD and FDD without the need for a guard interval or a guard band [5]. The idea is to allow transmissions in forward and reverse links to take place within an OFDM symbol simultaneously. Orthogonality is maintained since a subcarrier is assigned for a transmission in one direction only. The OFDM symbol in MDD occupies the whole frequency band. Therefore, MDD enjoys frequency diversity similar to that of TDD. In addition, MDD achieves full duplex transmission similar to that of FDD.

In OFDM/OFDMA, resource allocation has been a crucial issue in order to achieve the most efficient transmission. There have been tremendous works on resource allocation for OFDM/OFDMA since the works in [6], [7]. Several extensions to cooperative and relay systems have been found, e.g., in [8][10]. The standard method for resource allocation is to solve the optimization in the dual domain where optimization of the dual problem is separable into several subproblems. Lagrange multipliers in the dual problem are updated iteratively until all the constraints are satisfied. Solving the problem in the dual domain avoids exponential complexity, normally occurring in optimal subchannel assignment done by exhaustive search, in the original optimization problem.

In this paper, we extend the work on MDD in [5] to M2M communication systems and consider a resource allocation problem. The objective is to maximize the sum rate of the network under either total or individual power constraint. Optimization with the dual problem is proposed to solve the joint subcarrier and power allocation. The constraint of subchannel assignment in this paper is different from that in [5], where the subcarriers in a forward or reverse link are interleaved at regular spacing. Here, we relax such constraint to simplify the optimization and to achieve the best possible transmission rate.

The paper is organized as follows. Section II describes the system model. Section III describes MDD for M2M communications. Section IV derives resource allocation and proposes the algorithm. Section V discusses TDD and TDDOFDMA for comparison in the simulation. Section VI presents simulation results and discussions. Conclusions are given in Section VII. 


\section{SySTEM MODEL}

We consider a M2M system consisting of three terminals transmitting to each other. Generalization to more than three terminals is straightforward. Each terminal always has information to send. All terminals are sharing the same frequency band. However, on each subcarrier, only one transmitter can be active. The goal is to deliver information from one terminal to the other two terminals in the most efficient manner. Denote the terminal as $T_{m}, m=1,2,3$. We assume that the terminals are perfectly synchronized and have equal priority. Different priorities among terminals can be easily extended by giving different weights for different terminals in the objective function to be optimized.

A channel between a pair of terminals is assumed to be frequency selective fading and is characterized by the number of taps and power delay profile (PDP). Each tap experiences independent Rayleigh fading and sum of the average power of all taps is unity. The channel is assumed to follow exponential PDP, often considered in the literature. In a multicarrier system, the channel is normally considered in the frequency domain and is generated from conventional OFDM channel generation, i.e., taking $N$-point discrete Fourier transform of the frequency selective discrete-time channel, where $N$ is the number of subcarriers. Denote the channel gain from terminal $m$ to terminal $n$ on the subcarrier $k$ as $h_{m n}^{k}$. For the transmission from $T_{m}$ to $T_{n}, h_{m n}^{k}$ is considered to be a downlink channel for $T_{m}$ and an uplink channel for $T_{n}$ on subcarrier $k$. The channels between different terminal pairs are assumed to be independent and identically distributed. This could reflect the scenario where transmitter-receiver pairs have the same distance and similar propagation environment but they are not co-located. We assume the channels to be static during one packet duration and change independently in the next packet duration (i.e., quasi-static channel). Noise is assumed to be subsumed in the subcarrier SNR which is determined by the channel gain. Therefore, the $k$ th subcarrier SNR is determined by $\gamma_{m n}^{k}=\left|h_{m n}^{k}\right|^{2}$ whose variance determines the average SNR. Note that for a reciprocal channel, we have the same uplink and downlink channel gains, which mean $h_{m n}^{k}=h_{n m}^{k}$ and $\gamma_{m n}^{k}=\gamma_{n m}^{k}$.

\section{MulticARrier Division DUPLEXING (MDD)}

The key idea of MDD is to allow uplink and downlink transmissions on the same OFDM symbol simultaneously. The uplink and downlink transmissions occupy different subcarriers to maintain orthogonality. Detailed practical implementation of MDD has been discussed in [5] and will not be repeated here. For M2M systems, one subcarrier in an OFDM symbol can be assigned to any transmitter-receiver pair. In addition, we also allow one transmitter to transmit to more than one receiver and one receiver to receive from more than one transmitter in the same OFDM symbol. For a three-terminal system, at each subcarrier, there are totally nine possible transmitter-receiver configurations: $T_{1} \rightarrow T_{2}, T_{1} \rightarrow T_{3}, T_{2} \rightarrow T_{1}, T_{2} \rightarrow T_{3}, T_{3} \rightarrow$ $T_{1}, T_{3} \rightarrow T_{2}, T_{1} \rightarrow\left(T_{2}, T_{3}\right), T_{2} \rightarrow\left(T_{1}, T_{3}\right), T_{3} \rightarrow\left(T_{1}, T_{2}\right)$. At one subcarrier, one of the nine configurations is assigned.
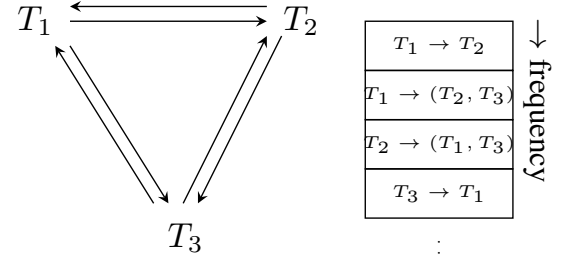

Fig. 1. Three-terminal system and channel assignment

We refer to the transmitter-receiver configuration assignment to each subcarrier as channel assignment. This is illustrated in Fig. 1 as an example.

The maximum transmission rate of a configuration is determined by Shannon capacity. Denote $P_{m}^{k}$ as the transmit power of terminal $m$ on subcarrier $k$. For a single transmitter and a single receiver, the transmission rate is the Shannon capacity of the corresponding link. More precisely, the rate of the configuration $T_{m} \rightarrow T_{n}$ on subcarrier $k$ is $\log _{2}\left(1+P_{m}^{k} \gamma_{m n}^{k}\right)$. For a configuration that has two receivers, the transmission rate must be limited by the channel of the weaker link to ensure successful decoding. Specifically, the rate of the configuration $T_{m} \rightarrow\left(T_{n}, T_{p}\right)$ on subcarrier $k$ is $2 \log _{2}\left(1+P_{m}^{k} \min \left(\gamma_{m n}^{k}, \gamma_{m p}^{k}\right)\right)$. The factor of two indicates double transmission rate from a single transmission to two receivers simultaneously.

\section{JoInt SubCARrier AND POWER AllocAtion FOR MDD}

Resource allocation is performed over the duration where the channel is static. There is no resource allocation across different packet intervals. The problem is to allocate subcarriers and transmission powers to achieve maximum sum rate of all terminals during one packet duration under a transmit power constraint. For each subcarrier, the algorithm needs to perform channel assignment and determine the transmit power.

Two types of constraints will be considered separately. First, total power constraint limits the total transmit power from all terminals. Second, individual power constraint restricts the transmit power of each terminal.

\section{A. Optimization Problem}

For convenience in the derivation, the function $\log _{2}(\cdot)$ will be replaced by a natural $\log (\cdot)$ in the following. Let $\phi_{k}$ denote a channel assignment for the subcarrier $k$,

$$
\phi_{k} \in\left\{T_{1} \rightarrow T_{2}, T_{1} \rightarrow T_{3}, \ldots, T_{3} \rightarrow\left(T_{1}, T_{2}\right)\right\}
$$

Denote the set of channel assignment as $\phi=\left\{\phi_{k} \mid k=\right.$ $0,1, \ldots, N-1\}$. Also, denote the set of allocated power as $\boldsymbol{P}=\left\{P_{m}^{k} \mid m\right.$ is the transmitter in $\left.\phi_{k} ; k=0,1, \ldots, N-1\right\}$.

The objective function is written as

$$
\max _{\boldsymbol{P}, \boldsymbol{\phi}} \sum_{k=0}^{N-1} \alpha_{k} \log \left(1+P_{m}^{k} \tilde{\gamma}_{k}\right),
$$


where $\alpha_{k}, \tilde{\gamma}_{k}$ are functions of $\phi_{k}$, determined by

$$
\alpha_{k}=\left\{\begin{array}{lll}
1, & \phi_{k} & \text { has one receiver } \\
2, & \phi_{k} & \text { has two receivers }
\end{array}\right.
$$

and

$$
\tilde{\gamma}_{k}=\left\{\begin{array}{l}
\gamma_{m n}^{k}, \quad \phi_{k}=T_{m} \rightarrow T_{n} \\
\min \left(\gamma_{m n}^{k}, \gamma_{m p}^{k}\right), \quad \phi_{k}=T_{m} \rightarrow\left(T_{n}, T_{p}\right)
\end{array}\right.
$$

The total power constraint can be written as

$$
\sum_{m=1}^{3} \sum_{k=0}^{N-1} P_{m}^{k} \leq P_{t}
$$

where $P_{t}$ is the total transmit power from all terminals and all subcarriers. The individual power constraint is written as

$$
\sum_{k=0}^{N-1} P_{m}^{k} \leq P_{s}, m=1,2,3
$$

where $P_{s}$ is the total transmit power of all subcarriers at each terminal, assumed to be the equal for all terminals. Naturally, the power constraint also has to satisfy

$$
P_{m}^{k} \geq 0, \quad \forall m, k
$$

Our optimization problem is thus (2) subject to (5) or (6) and (7). It is a difficult nonconvex optimization problem due to its combinatorial structure in choosing $\phi$. Nevertheless, we can apply the standard approach in resource allocation for OFDMA to solve the optimization problem efficiently in the dual domain [7].

\section{B. Optimization via Dual Problem}

First, we consider the problem with total power constraint. Define the Lagrangian to be

$\mathcal{L}(\boldsymbol{P}, \boldsymbol{\phi}, \lambda)=\sum_{k=0}^{N-1} \alpha_{k} \log \left(1+P_{m}^{k} \tilde{\gamma}_{k}\right)-\lambda\left(\sum_{m=1}^{3} \sum_{k=0}^{N-1} P_{m}^{k}-P_{t}\right)$,

where $\lambda$ is a Lagrange multiplier associated with the total power constraint. The dual function is thus

$$
\begin{aligned}
\qquad(\lambda)= & \max _{\boldsymbol{P}, \boldsymbol{\phi}} \mathcal{L}(\boldsymbol{P}, \boldsymbol{\phi}, \lambda) \\
\text { subject to } & P_{m}^{k} \geq 0, \quad \forall m, k .
\end{aligned}
$$

Given a fixed $\phi$, to find the optimal power $P_{m}^{k *}$, (9) can be decomposed into $N$ independent subproblems:

$$
\max _{P_{m}^{k} \geq 0} \alpha_{k} \log \left(1+P_{m}^{k} \tilde{\gamma}_{k}\right)-\lambda P_{m}^{k}
$$

Take the derivative of the object in (10) and set it to zero, the optimal $P_{m}^{k *}$ is determined as

$$
P_{m}^{k *}=\left[\frac{\alpha_{k}}{\lambda}-\frac{1}{\tilde{\gamma}_{k}}\right]^{+}
$$

where $[x]^{+}$is $\max (0, x)$. Substituting (11) back to the object in (10), the algorithm searches for the optimal $\phi_{k}^{*}$, which yields corresponding $\alpha_{k}$ and $\tilde{\gamma}_{k}$, that maximize the object in (10):

$$
\phi_{k}^{*}=\underset{\phi_{k}}{\operatorname{argmax}} \alpha_{k} \log \left(1+\left[\frac{\alpha_{k}}{\lambda}-\frac{1}{\tilde{\gamma}_{k}}\right]^{+} \tilde{\gamma}_{k}\right)-\lambda\left[\frac{\alpha_{k}}{\lambda}-\frac{1}{\tilde{\gamma}_{k}}\right]^{+} .
$$

To find the solution, the algorithm determines $\lambda$ that minimizes the dual function,

$$
\min _{\lambda} g(\lambda)
$$

This can be done by updating $\lambda$ until the total power constraint is satisfied. Bisection method can be applied to find the optimal $\lambda$ efficiently.

For the problem with individual power constraint, similar steps can be followed for derivation. However, the algorithm is more complicated as there are now three constraints to satisfy. Define the Lagrangian to be

$\mathcal{L}(\boldsymbol{P}, \boldsymbol{\phi}, \boldsymbol{\lambda})=\sum_{k=0}^{N-1} \alpha_{k} \log \left(1+P_{m}^{k} \tilde{\gamma}_{k}\right)-\sum_{m=1}^{3} \lambda_{m}\left(\sum_{k=0}^{N-1} P_{m}^{k}-P_{s}\right)$,

where $\lambda_{m}$ is the Lagrange multiplier associated with power constraint of terminal $m, \boldsymbol{\lambda}=\left\{\lambda_{1}, \lambda_{2}, \lambda_{3}\right\}$. The dual function $g(\boldsymbol{\lambda})$ is similar to (9) with the Lagrangian replaced by (14). The optimal power $P_{m}^{k *}$ is of the form

$$
P_{m}^{k *}=\left[\frac{\alpha_{k}}{\lambda_{m}}-\frac{1}{\tilde{\gamma}_{k}}\right]^{+}
$$

and the optimal $\phi_{k}^{*}$ is

$\phi_{k}^{*}=\underset{\phi_{k}}{\operatorname{argmax}} \alpha_{k} \log \left(1+\left[\frac{\alpha_{k}}{\lambda_{m}}-\frac{1}{\tilde{\gamma}_{k}}\right]^{+} \tilde{\gamma}_{k}\right)-\lambda_{m}\left[\frac{\alpha_{k}}{\lambda_{m}}-\frac{1}{\tilde{\gamma}_{k}}\right]^{+}$.

In (16), $\lambda_{m}$ corresponds to the power constraint of the transmitter indicated in $\phi_{k}$. The solution is obtained with

$$
\min _{\boldsymbol{\lambda}} g(\boldsymbol{\lambda})
$$

via an algorithm such as the subgradient method [11] or the ellipsoid method [12], that updates $\boldsymbol{\lambda}$ to satisfy the power constraint in (6).

Proposition 1: For the dual problem with the individual power constraint, the subgradient for $g(\boldsymbol{\lambda})$ is

$$
\boldsymbol{\theta}=\boldsymbol{P}_{\boldsymbol{s}}-\sum_{k=1}^{N} \boldsymbol{P}^{k *}
$$

where $\boldsymbol{P}_{\boldsymbol{s}}=\left[\begin{array}{lll}P_{s} & P_{s} & P_{s}\end{array}\right]^{t}$, and $\boldsymbol{P}^{k *}=\left[\begin{array}{lll}P_{1}^{k *} & P_{2}^{k *} & P_{3}^{k *}\end{array}\right]^{t}$ optimizing $g(\boldsymbol{\lambda})$.

Proof: Similar to [6].

For completeness, we summarize the algorithm for joint subcarrier and power allocation for MDD in the Algorithm 1 .

To aid in the initialization of $\boldsymbol{\lambda}$ in the individual power constraint problem, the following proposition is derived. 


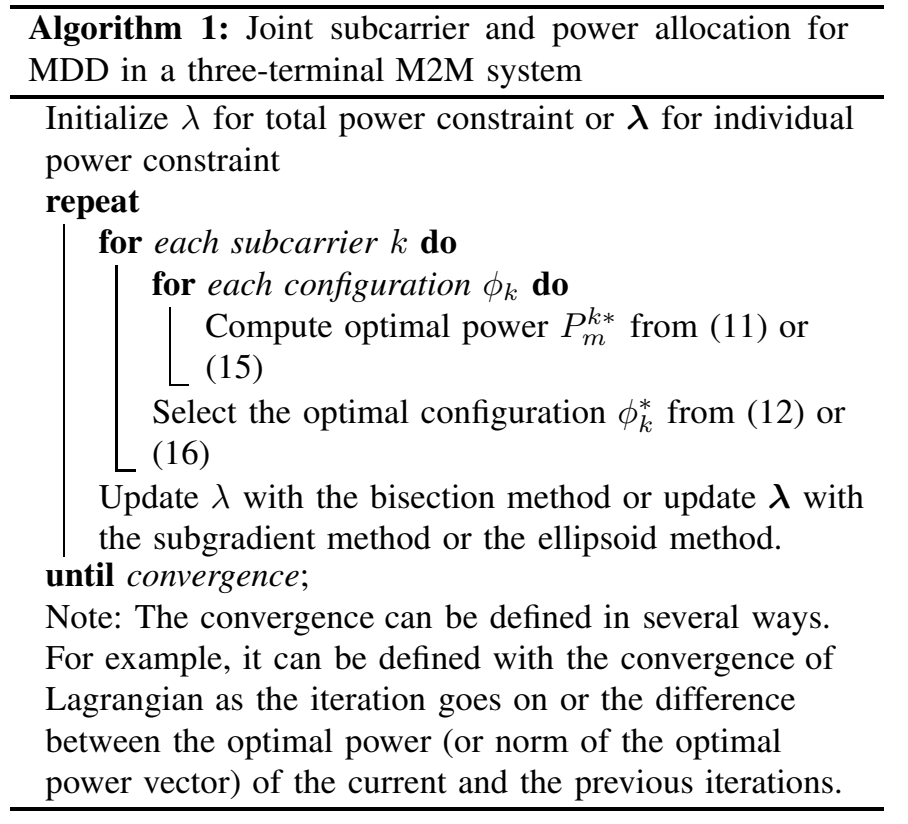

Proposition 2: Let $\lambda_{m}^{*}, m=1,2,3$, be the optimized variables of (17). Then,

$$
\min _{k} \min _{\substack{\phi_{k}: T_{m} \text { is the } \\ \text { transmitter }}}\left(\frac{\alpha_{k}}{P_{s}+\frac{1}{\tilde{\gamma}_{k}}}\right) \leq \lambda_{m}^{*} \leq \frac{2 N}{P_{s}} .
$$

Proof: Suppose at least one subcarrier $k^{\prime}$ is assigned to terminal $m$ at the optimal solution of (17). Then, at the optimum, using the individual power constraint, we can show that

$$
P_{s}=\sum_{k=0}^{N-1}\left[\frac{\alpha_{k}}{\lambda_{m}^{*}}-\frac{1}{\tilde{\gamma_{k}}}\right]^{+} \geq \frac{\alpha_{k^{\prime}}}{\lambda_{m}^{*}}-\frac{1}{\tilde{\gamma}_{k^{\prime}}} .
$$

Therefore,

$$
\lambda_{m}^{*} \geq \frac{\alpha_{k^{\prime}}}{P_{s}+\frac{1}{\tilde{\gamma}_{k^{\prime}}}} \geq \min _{k} \min _{\substack{\phi_{k}: T_{m} \text { is the } \\ \text { transmitter }}}\left(\frac{\alpha_{k}}{P_{s}+\frac{1}{\tilde{\gamma}_{k}}}\right),
$$

and we have the lower bound. In addition, since

$$
P_{s}=\sum_{k=0}^{N-1}\left[\frac{\alpha_{k}}{\lambda_{m}^{*}}-\frac{1}{\tilde{\gamma_{k}}}\right]^{+} \leq \sum_{k=0}^{N-1} \frac{\alpha_{k}}{\lambda_{m}^{*}} \leq \frac{2 N}{\lambda_{m}^{*}},
$$

we have the upper bound $\lambda_{m}^{*} \leq \frac{2 N}{P_{s}}$.

Proposition 2 can be easily applied to the total power constraint, which results in the following.

Proposition 3: Let $\lambda^{*}$ be the optimized variable of (13). Then,

$$
\min _{k} \min _{\phi_{k}}\left(\frac{\alpha_{k}}{P_{t}+\frac{1}{\tilde{\gamma}_{k}}}\right) \leq \lambda^{*} \leq \frac{2 N}{P_{t}} .
$$

Proof: Similar to Proposition 2, replace $P_{s}$ with $P_{t}$ and consider that any terminal is a possible transmitter.

The proposed algorithm is a centralized version and needs the knowledge of all channels. Therefore, all the terminals have to feedback the channel information to a central entity which could be one of the terminals acting as a master or a dedicated entity whose function is the subcarrier and power allocation. Once the optimization is done, the central entity has to inform resulting allocation to all terminals. These procedures could be part of initial setup stage of the M2M communications, which is beyond the scope of this paper.

Note that in reciprocal channels, there exists more than one optimal $\phi_{k}$ at each subcarrier. In such case, arbitrary tie-breaking is performed by randomly choosing one of the optimal configurations.

\section{Complexity}

Let the total number of possible transmitter-receiver configurations be $C$. For a three-terminal M2M system, the number of configurations is nine. More generally, for $M$-terminal M2M systems, transmitting to more than two receivers simultaneously is possible. In this case, the total number of configurations is increased to $M\left(2^{M-1}-1\right)$. The proposed resource allocation reduces the complexity from $\mathcal{O}\left(C^{N}\right)$ in exhaustive search for the optimal configuration to $\mathcal{O}(C N)$ due to separability of the Lagrangian in the dual domain.

For reciprocal channels, the complexity can be reduced a bit further. For example, $T_{1} \rightarrow T_{2}$ achieves the same rate as $T_{2} \rightarrow T_{1}$. Then, the algorithm needs to work only with the channel between them regardless of the direction. In the threeterminal system, $C$ is reduced to six.

Note that although the algorithm has a polynomial complexity with $N$, it still has exponential complexity with $M$. To further reduce complexity in practice, the algorithm may be modified to operate on a subchannel consisting of adjacent subcarriers with approximately similar channel gains rather than on individual subcarriers. Such approach has been considered in OFDMA resource allocation, e.g., in [13], [14].

\section{TDD AND TDD-OFDMA SCHEMES}

In multicarrier M2M systems, we will compare the performance of MDD with two conventional schemes: TDD and TDD-OFDMA. In TDD and TDD-OFDMA, each terminal is able to only transmit or receive a signal during an OFDM symbol. Consider a three-terminal system, the following configurations can occur.

- TDD: During one packet interval, there is only one transmitter while there can be one or two receivers.

- TDD-OFDMA: During one packet interval, in addition to the TDD scheme, there can be two transmitters sharing the same OFDM symbol but they transmit at different subcarriers to the same another terminal which is not transmitting.

\section{A. Three Terminals under Channel Reciprocity}

For three terminals operating on channels with reciprocity, it can be shown that TDD-OFDMA does not provide any advantages to TDD. Without loss of generality, suppose we consider TDD-OFDMA where $T_{1}$ is a transmitter or a receiver for a given subcarrier as shown in Fig. 2. Given the transmit power $P$, when $T_{1}$ is the transmitter, the rate of this subcarrier 


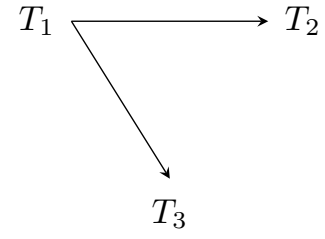

(a)

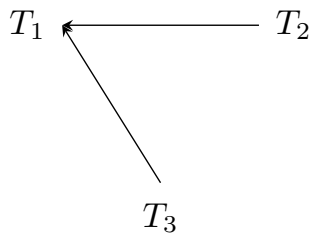

(b)
Fig. 2. TDD-OFDMA (a) $T_{1}$ is the transmitter. Either $T_{2}$ or $T_{3}$ is a receiver on one subcarrier. (b) $T_{1}$ is the receiver. Only one of $T_{2}$ or $T_{3}$ is the transmitter on one subcarrier.

is the maximum between $2 \log _{2}\left(1+P \min \left(\gamma_{12}, \gamma_{13}\right)\right)$ and $\log _{2}\left(1+P \max \left(\gamma_{12}, \gamma_{13}\right)\right)$. When $T_{1}$ is the receiver, the rate of this subcarrier can be either $\log _{2}\left(1+P \gamma_{21}\right)$ or $\log _{2}\left(1+P \gamma_{31}\right)$. When channel reciprocity holds, $\gamma_{12}=\gamma_{21}$ and $\gamma_{13}=\gamma_{31}$, we can see that the rate obtained when $T_{1}$ is the transmitter is at least the rate obtained when $T_{1}$ is the receiver. Therefore, the configuration of two transmitters and one receiver during one OFDM symbol does not provide any advantages in this case. TDD-OFDMA is equivalent to TDD for the case of three terminals with channel reciprocity.

\section{RESULTS AND DisCUSSIONS}

The performance of MDD is evaluated by simulation. The average sum rate is computed by the sum rate averaged over the number of subcarriers and different channel realizations. It is plotted against the average SNR on the x-axis. The number of subcarriers is set to $N=256$. Under total power constraint, the total transmit power is equal to the number of subcarriers, $P_{t}=N$. Under individual power constraint, the transmit power of each terminal is the total transmit power divided by three, $P_{s}=N / 3$, to make a fair comparison with the total power constraint. We assume channel reciprocity to hold under the considered system.

Fig. 3 illustrates the performance of joint subcarrier and power allocation of the three schemes: MDD, TDD and TDDOFDMA. The channel is assumed to be 16-tap. Individual power constraint is applied. As explained in Section V, the performance of TDD and TDD-OFDMA is shown to be the same. Each user achieves similar average rate and is about one third of the average sum rate for all multiplexing schemes since we do not consider any priorities among users. A larger gain of MDD over TDD, TDD-OFDMA is visible at a larger SNR, where diversity of MDD shows a larger effect. About 1.3-dB gain over TDD and TDD-OFDMA is achieved with MDD at the average sum rate of $14 \mathrm{bit} / \mathrm{s} / \mathrm{Hz}$.

Fig. 4 compares average sum rate of MDD under total power constraint (TPC) and individual power constraint (IPC) with different types of resource allocation, namely joint subcarrier and power allocation (PA), separate PA, and no PA. Separate PA refers to the scheme where channel assignment is done first based on unit power assumed at each subcarrier and then power allocation is performed to satisfy respective power constraints given fixed earlier channel assignment. For

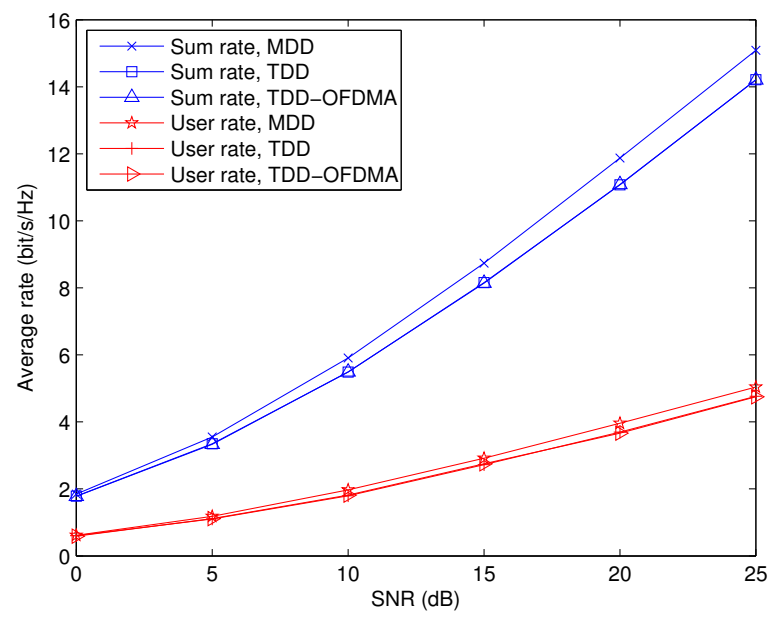

Fig. 3. Joint subcarrier and power allocation for MDD, TDD and TDDOFDMA on a 16-tap channel. User rate is the average rate of any terminal.

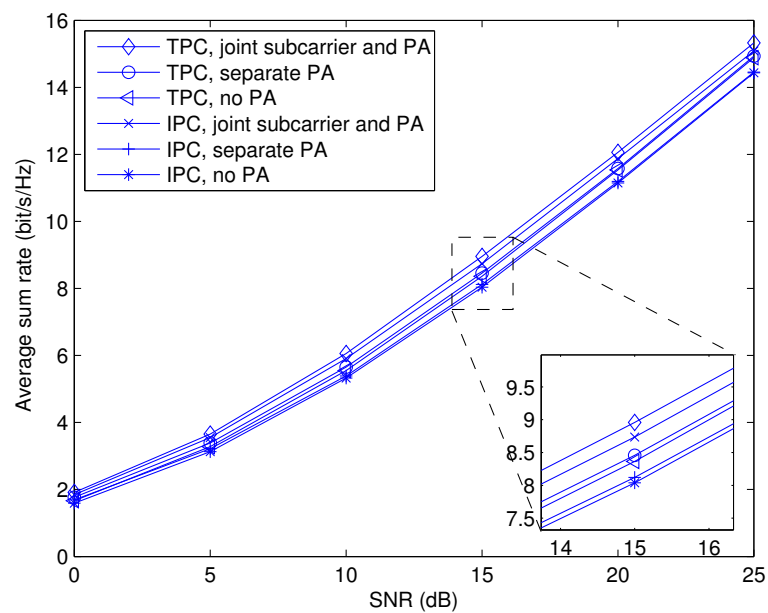

Fig. 4. Average sum rate of MDD under total power constraint (TPC) and individual power constraint (IPC). Comparison between joint subcarrier and power allocation (PA), separate PA and no PA.

channel assignment in separate PA, the transmitter-receiver configuration that achieves the maximum rate is chosen at each subcarrier assigned with unit power. No PA refers to the scheme that performs channel assignment alone without power allocation. Here, a 16-tap channel is assumed. With joint subcarrier and PA, as expected, the TPC outperforms IPC although the gain is small. It appears that separate PA for both TPC and IPC is suboptimal and has only very limited gain compared to No PA.

Fig. 5 shows average sum rate of MDD under IPC and the influence of the number of channel taps. It is expected that 4-tap channel will have lower average sum rate than 8-tap and 16-tap channels due to its less frequency diversity. The gap of the average sum rate is more distinct at the high SNR.

Fig. 6 shows the probability of transmitting to two receivers simultaneously in MDD. Obviously, most of the time, two 


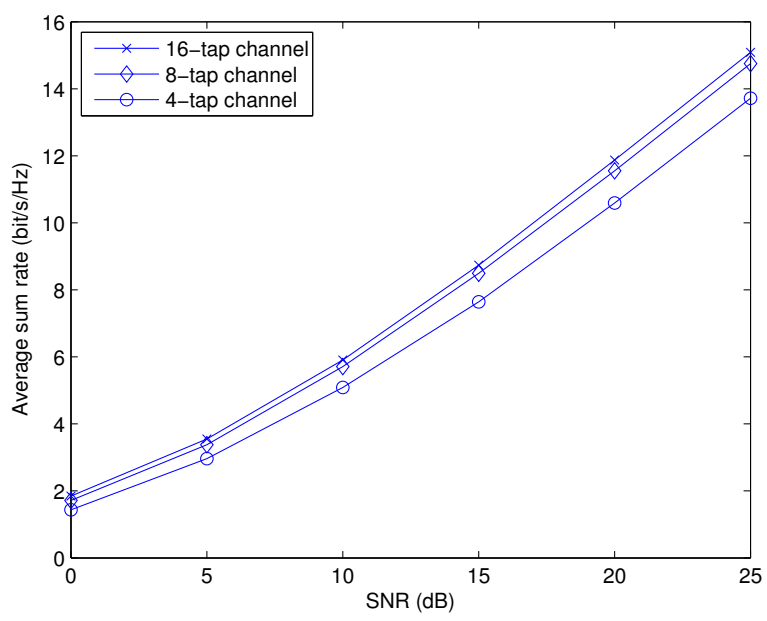

Fig. 5. Average sum rate of MDD under individual power constraint. Comparison among 4-tap, 8-tap and 16-tap channels.

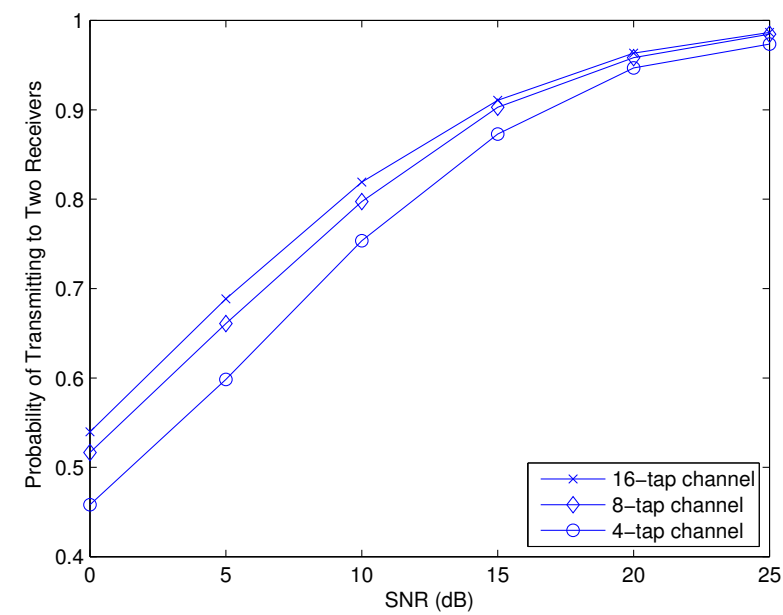

Fig. 6. Probability of transmitting to two receivers simultaneously in MDD. Comparison among 4-tap, 8-tap and 16-tap channels.

receivers are preferred especially when the SNR is high. Transmitting to two receivers is favorable in the channel with a high number of taps than the channel with a low number of taps for all SNRs.

Fig. 7 shows the convergence of the proposed joint subcarrier and power allocation of MDD with individual power constraint. The channel condition is 16-tap with SNR equal to $10 \mathrm{~dB}$. The algorithm to update $\boldsymbol{\lambda}$ is the ellipsoid method. We can see that the algorithm converges fast for all ten exemplary channel realizations.

\section{CONCLUSIONS}

This paper considers joint subcarrier and power allocation for M2M MDD systems. The objective is sum rate maximization of the network under either total or individual power constraint of the terminals. Optimization is solved by the standard algorithm for OFDMA resource allocation in the

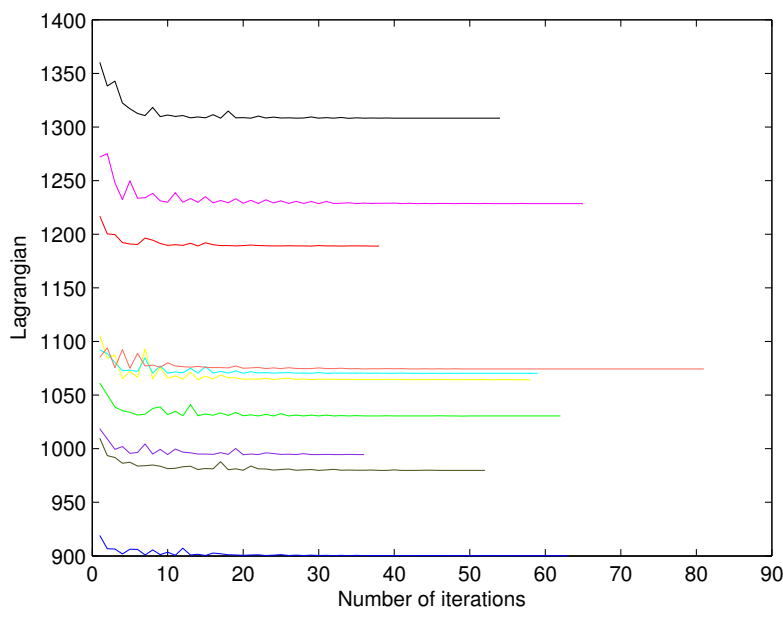

Fig. 7. Plot of Lagrangian, $g(\boldsymbol{\lambda})$, with an ellipsoid method, for ten exemplary channel realizations. The channel is 16-tap with SNR equal to $10 \mathrm{~dB}$.

dual domain. The main findings given by simulation are the followings: MDD outperforms TDD and TDD-OFDMA in a three-terminal system, MDD exploits frequency diversity efficiently and the proposed algorithm converges quickly to the solution.

\section{REFERENCES}

[1] A. Vakali and G. Pallis, "Content delivery networks: status and trends," IEEE Internet Computing, vol. 7, pp. 68-74, Nov. 2003.

[2] E. K. Lua, J. Crowcroft, M. Pias, R. Sharma, and S. Lim, "A survey and comparison of peer-to-peer overlay network schemes," IEEE Comm. Surveys and Tutorials, vol. 7, no. 2, pp. 72-93, 2005.

[3] L. Hanzo, Y. Akhtman, L. Wang and M. Jiang, MIMO-OFDM for LTE, WiFi and WiMAX: Coherent versus Non-coherent and Cooperative Turbo Transceivers, Wiley-IEEE Press, 2010.

[4] T. S. Rappaport, Wireless Communications: Principles and Practice, Prentice-Hall, 2nd Edition, 2002.

[5] W. Qiu and H. Minn, "Orthogonal multicarrier division duplexing for point-to-point communications," to appear in Proc. IEEE International Conference on Communications (ICC), June 2012.

[6] W. Yu and R. Lui, "Dual methods for nonconvex spectrum optmization of multicarrier systems," IEEE Trans. Commun., vol. 54, pp. 1310-1322, July 2006.

[7] K. Seong, M. Mohseni and J. M. Cioffi, "Optimal resource allocation for OFDMA downlink systems," in Proc. Int. Symp. on Inform. Theory (ISIT), pp. 1394-1398, 9-14 July 2006.

[8] T. C.-Y. Ng and W. Yu, "Joint optimization of relay strategies and resource allocations in cooperative cellular networks," J. Select. Areas Commun. vol. 25, pp. 328-339, Feb. 2007.

[9] Z. Han, T. Himsoon, W. Siriwongpairat, and K. Liu, "Resource allocation for multiuser cooperative OFDM networks: Who helps whom and how to cooperate," IEEE Trans. Veh. Tech., vol. 58, pp. 2378-2391, June 2009.

[10] W. Wang and R. Wu, "Capacity maximization for OFDM two-hop relay system with separate power constraints," IEEE Trans. Veh. Tech., vol. 58 , pp. 4943-4954, Nov. 2009.

[11] N. Z. Shor, Minimization Methods for Non-differentiable Functions, Springer Series in Computational Mathematics. Springer, 1985.

[12] R. G. Bland, D. Goldfarb, and M. J. Todd, "The ellipsoid method: A survey," Operation Research, 29(6), pp. 1039-1091, 1981.

[13] V. D. Papoutsis and S. A. Kotsopoulos, "Chunk-based resource allocation in distributed MISO-OFDMA systems with fairness guarantee," IEEE Commun. Lett., vol. 15, pp. 377-379, Apr. 2011.

[14] H. Zhu and J. Wang, "Chunk-based resource allocation in OFDMA systems-part I: chunk allocation," IEEE Trans. Commun., vol. 57, pp. 2734-2744, Sep. 2009. 